\title{
Ethical and Psychosocial Impact of Female Infertility
}

\author{
Ophra Leyser-Whalen • Jeff R. Temple • \\ John Y. Phelps
}

Published online: 2 September 2012

(C) Springer Science+Business Media, LLC 2012

\begin{abstract}
This manuscript reviews research from the past year on the ethical and psychosocial impact of infertility on women and men. We discuss several issues surrounding ovarian stimulation, particularly high-order multiple births, egg banking (especially for research purposes), and diminished ovarian reserve. We also present recent work on distress and counseling, which includes greater attention to subgroups of infertile women. More research on issues confronting men has emerged recently, and we outline these with regard to their relationships with infertile women, or as the infertility patient. Last, we outline some ethical issues posed by newer procedures of fertility preservation and uterine transplant.
\end{abstract}

Keywords Infertility · Fertility treatment · Ethics · Psychosocial factors $\cdot$ Female $\cdot$ Male $\cdot$ Female infertility . Male infertility $\cdot$ Egg banking $\cdot$ Ovarian stimulation . High-order multiple births · Diminished ovarian reserve . Uterine transplant $\cdot$ Fertility preservation $\cdot$ Assisted reproductive medicine

\section{Introduction}

As practitioners managing patients with infertility, we must be aware of ongoing ethical issues. An interview study with 22 infertility physicians in the United Kingdom about their

O. Leyser-Whalen

Department of Sociology and Anthropology,

The University of Texas at El Paso,

Old Main Bldg.,

El Paso, TX 79968, USA

J. R. Temple $\cdot$ J. Y. Phelps $(\bowtie)$

Department of Obstetrics and Gynecology, UTMB Health,

301 University Blvd,

Galveston, TX 77555-0587, USA

e-mail: jyphelps@utmb.edu views of the ethics in their work reported that many ethical issues generally existed below the surface and became normalized as routine practice. These issues differed by clinic, denoting a certain "clinic ethos," where practitioners explicitly or implicitly agreed on issues. What is seen as ethically appropriate or controversial, however, has changed over time [1]. With this in mind, clinicians, researchers, and bioethicists must repeatedly examine the ethical issues surrounding infertility.

Furthermore, to ensure comprehensive care, we must attend to the psychosocial processes inherent in infertility and fertility treatments. Attention to these issues not only provides ethical treatment for a patient but also may be important for clinic success. For example, findings suggest that baseline anxiety and depression may negatively influence the in vitro fertilization (IVF) clinical pregnancy rate in women with tubal factor infertility. The researchers also found that higher levels of norepinephrine, a biological marker for stress, was negatively associated with the percentage of good quality embryos, yet not associated with self-reported anxiety or depression [2].

These two studies are among the recent literature on ethics and psychosocial aspects of infertility that this paper reviews. After outlining our methodology, we discuss several issues surrounding ovarian stimulation, distress and counseling, and specific issues confronting men. Last, we outline research on newer procedures of fertility preservation and uterine transplant (UTx).

\section{Methods}

We conducted PubMed and Google Scholar searches for articles published between the years 2011 and 2012. Search terms included "infertility" in addition to the following: "psychosocial"; "psychological"; "sociology"; or "ethics." 
Articles also were found from the reference lists of the manuscripts identified through these searches.

\section{Ovarian Stimulation}

Ethical issues still surround ovarian stimulation [3], especially on the topics of higher-order multiple births (HOMB), egg banking, and diminished ovarian reserve.

\section{Higher-Order Multiple Births}

Between 1997 and 2009, the use of IVF has increased $84 \%$, yet the number of embryos transferred and multiple pregnancies, have decreased [4]. However, several scholars believe that physicians should remain concerned with multiple pregnancy rates due to the many risks to both infants and the mother, as well as the high medical costs associated with delivery, neonatal care, and long-term complications of HOMB $[4,5,6 \bullet]$.

Ethical issues surrounding HOMB often revolve around the principle of parental responsibility, which posits that people should not be only concerned with their own interests in reproducing, but also the welfare of the child [6 $6^{\circ}$. This can be applied to potential or existing children; thus, an assessment of the resources available to the parent(s) for both obtaining and caring for children enter into this equation. Manninen [6•] implicates various people and entities that should be held ethically accountable for HOMB, which include the patient, clinic, media that glamorizes HOMB, and insurance companies that do not cover certain fertility treatments and thus drive people to utilize fewer and riskier attempts at assisted reproduction. The author also cites studies that show that paying for more fertility treatment options may actually be more cost effective for insurers than paying for peri- and post-natal care of HOMB.

For fertility physicians, Manninen [6•] suggests policies that extend beyond simply educating patients but create official guidelines on the number of embryos transferred for IVF and reduce the chances for HOMB during intrauterine insemination. Similar requests have been made of the Centers for Disease Control and Prevention and the American Society for Reproductive Medicine (ASRM) to impose stricter regulations on both fertility providers and insurance companies to favor safer IVF techniques [5].

Critics of regulation worry that restrictions may limit the reproductive autonomy of infertile patients, and that decisions on the number of embryo transfers should not only remain with the patient and practitioner, but should be based on patient age and number of high-quality embryos [7]. Others have argued that adopting certain regulations that decrease the chances of an infertile couple successfully conceiving in a particular treatment cycle does not strip an infertile couple of their capacity to procreate if they are given future access to treatment. To ensure this, insurance companies should cover repeated cycles. Furthermore, in order to lessen a woman's exposure to repeated invasive procedures, she can freeze procured eggs to limit retrievals or decrease the potency of ovulation-inducing drugs [6•].

Additional suggestions to limit HOMB include continued research into improved monitoring, advanced culture techniques, enhanced implantation rates, and tests to predict an embryo's genetic and developmental competence [4]. Others propose limitations of one or two embryo transfers, which is more obtainable due to new techniques, such as single embryo transfer, which is the standard in some European countries [8].

\section{Egg Banking}

The health risk for women donating oocytes for somatic cell nuclear transfer (SCNT) has been an indirect argument against embryonic stem cell research. One such risk is ovarian hyperstimulation syndrome (OHSS), which some say women are not adequately warned about during the consent process [9]. Others, however, have argued that incidence rates of OHSS are not applicable to oocyte donors, especially if proper precautions are taken. New developments may help to defuse this debate [10]. First, new stimulation protocols, such as replacing human chorionic gonadotrophin (hCG) with hLH or gonadotropin-releasing hormone (GnRH) for women who do not have PCOS, has been suggested to reduce the risk of OHSS [11, 12]. Second, "spare" oocytes may soon be available, negating the need to stimulate women solely for research purposes. With vitrification (ultrarapid freezing) and improvements to slow-freezing methods, freezing oocytes is becoming more efficient. If oocytes, rather than embryos, are frozen, this should deter ethical problems for both patients and policymakers who attribute a high moral value to the embryo [12].

With the creation of a limited number of embryos for each cycle, few will become supernumerary. First, this will help lessen couple-level conflicts surrounding embryo disposition if a disagreement arises (e.g., divorce), because the woman will be the sole decision maker regarding her oocytes. Second, it is likely that when given a choice regarding the outcome of unused genetic materials (destruction, donation for science, or donation for reproduction), many women will donate to science due to fewer ethical and psychological ramifications [12]. Moreover, Mertes and Pennings [12] contend that this new freezing method will result in increased "social freezing," where women bank their oocytes until a time when they are ready to reproduce. The oocytes of women who have not found a partner by the time they reach the age limit could be donated to science, as could the oocytes from "medical freezers."

This two-phased donation cycle of stimulation and pickup, followed by donation, could avoid the ethical questions 
of donors being lured into taking unnecessary risks for a study with uncertain benefits [12]. Mertes and Pennings [12] also suggest a temporary solution of an egg-sharing program whereby social freezers donate some of their oocytes at the time of retrieval, in exchange for a participation in the costs related to egg retrieval. They contend that this is less controversial than egg sharing for immediate reproductive purposes.

Last, some believe that women who donate oocytes for research purposes should be compensated similarly to those who donate for reproductive purposes [11, 13]. Others warn about unregulated egg donor recruitment agencies, the necessity for enforced parameters that ensure nonexploitive recruitment tactics, patient consent, and development of a registry of donors to follow-up adequately on their health [13-16].

\section{Diminished Ovarian Reserve}

Karipcin and colleagues [17] maintain that physicians can legally and ethically decline ovulation induction to patients with diminished ovarian reserve when chances of successful pregnancy are futile. There is more ambiguity when patients have a minimal (but possible) chance of achieving pregnancy. For inducing ovulation for patients with diminished ovarian reserve, the authors suggest relying on the guidelines and ethics proposed by ASRM, as well as those of the American Medical Association (AMA) and American Congress of Obstetricians and Gynecologists. They further suggest that these patients should be counseled about their other options, such as donor eggs and adoption.

\section{Psychosocial Distress and Counseling}

During the past few years, more attention has been paid to the emotional distress associated with infertility and the process of assisted reproduction, including greater attention to subgroups of infertile women. For example, compared with white women, Asian, black, and Hispanic women had greater ethical concerns about infertility treatments and these concerns were associated with lower odds of getting tests and receiving treatment [18].

In other comparisons, infertile women who had never conceived had much higher levels of fertility-specific distress than infertile women who had been pregnant, irrespective of birth outcome. Furthermore, they were more likely to have seen a physician, had tests, and sought treatment. Although pregnancy loss is devastating, this study shows no significant differences of infertility-specific distress between women who had not had a live birth and women who had live births. The authors in this study suggest that the psychosocial primary/secondary infertility distinction is based on conception, rather than birth [19].
Other studies compared treatment versus no treatment groups. For example, women who did not seek fertility treatment placed less importance on the idea of parenthood, yet also reported lower levels of self-esteem and life satisfaction than women who sought treatment, regardless of treatment outcomes [20]. Similarly, Johnson and Fledderjohann [21] found that infertile women who had undergone treatment reported higher levels of distress than those who had not, irrespective of type of diagnosis.

Other research has found that the amount of social support that a patient receives can have significant effects on stress levels [22]. Compared with white and Asian women, black women were less likely to report encouragement for treatment from their partners and family members [18]. Greater social support can lead to less stress, with some levels mediated by different coping strategies [23].

With regard to infertility counseling, Blyth [24] attempts to map out what guidelines exist globally. In Australia, New Zealand, and the United Kingdom, counselors are actively engaged in defining their roles and scope. These countries also require specific academic and professional qualifications for psychosocial counselors in accredited fertility clinics. Australia, New Zealand, and some European countries necessitate counseling for specific types of fertility treatments, with most concentration on reproduction that involves a third party, which may include patients, donors, surrogates, and their partners.

Clinic personnel, however, may need to work harder to relay counseling standards and availability. In interviewing clinic workers, patients, and policy makers, Machin [25] found disparity in perceptions between clinic personnel and patients with their views of availability of counseling. Furthermore, the majority of those regulating and working in clinics were unaware of the difficulties that people had after completion of treatment, such as decisions about frozen embryos, which indicates that clinics may need to be proactive in providing ongoing support.

In other studies, physicians discussed the problem of trying to ensure the welfare of the child produced by any fertility treatment and felt that this was particularly difficult because of a lack of information on prospective parents and a lack of criteria for adequate parenting, although some areas of Australia and New Zealand require parents to pass criminal background checks. Physicians and regulators also are undecided on such issues as disclosing information on donated gametes to the child and whether infertility clinicians should be making such decisions at all [1, 24].

Other research has more specific suggestions for clinicians. For example, Frith et al. [1] found disagreement among the practitioners on the ethics of treating same-sex couples. Citing a recent lesbian discrimination suit, Storrow [26] argues that the AMA would benefit from strong nondiscrimination language in its physician-patient relationship 
provisions. Other suggestions for clinicians include more emphasis on couple-level issues, especially those of sexual health and intimacy, through the facilitation of information gathering and its analysis, help in decision-making, evaluation, support, and therapy [27]. Interventions designed to redirect goals and enhance coping skills also may prove viable $[23,28]$.

\section{Men and Male Infertility}

Although most infertility research focuses on women, one cannot omit men's roles. There is a small but growing literature on ethical and psychosocial aspects surrounding men and infertility, either analyzing men in their relationships with infertile women or as the infertility patient. One study focused on the hormonal levels and psychological symptoms in infertile men and found that testosterone level was inversely associated with anxiety, but FSH and LH were positively associated with anxiety. There was no association between anxiety and prolactin and estradiol [29].

In regard to ethical considerations, some note that when presented with an infertile couple, some physicians do not thoroughly examine the male partner and instead opt for the most advanced reproductive technology currently available, IVF/ICSI, to address both male and female factor infertility. These authors caution that this procedure may be overutilized and that a lack of proper evaluation of the male partner may not only overlook a correctable cause for male infertility but also may miss a serious medical condition, which could ultimately result in greater harm to the man or his potential offspring. Moreover, it has been suggested that failure to seek out male factor infertility limits the patient's ability to make informed choices about intervention and transfers the burden of his medical condition onto his female partner and child, who then bear the burden of potential risks associated with assisted reproductive technologies [30].

Posthumous sperm retrieval (PSR) presents another ethically controversial topic. PSR is not legal in several European countries, Canada, and Western Australia, and there are no clear guidelines for its use in the United States. Of particular concern are cases in which a man unexpectedly dies and his female partner requests the removal of sperm from his body for the purpose of future conception. This raises questions about the man's prior consent, autonomy, and bodily integrity. One suggestion has been to require a grieving period of at least 6 months for the widow before the saved sperm can be used [30].

Moreover, issues surrounding telling children if they were conceived with donor insemination (DI) still abound. Although recent studies advise parents and practitioners that children should be informed, parents tend to withhold this information [30]. Chubak and Thomas [30] contend that truth- telling is part of medicine and that children conceived through DI should be informed to avoid potential medical problems and emotional damage to their parental relationships.

Compared with women, there has been less research about the experiences of men affected by infertility, yet there is a growing body of literature from diverse settings using varied designs and data sources on the psychosocial experiences. Although few studies reported whether male-specific cutoff scores were used, findings indicate that diagnosis and treatment were associated with elevated infertility-specific anxiety, especially for men who were socially isolated or had an avoidant coping style. These rates, however, were lower than women's scores, and the overall prevalence of clinically significant mental health problems were no higher than those found in the general population. Unsuccessful treatment, however, could lead to long-term emotional consequences [31•].

Although additional research is needed, studies consistently show that infertile men in resource-constrained countries are faced with less accessible services, have more limited knowledge of reproduction and fertility, and are more reluctant to participate in the fertility treatment process, in part due to cultural beliefs [31•]. Among the U.S. population, there are still gaps in knowledge about proper counseling, seeking, persisting with, and deciding to cease treatment, experiences of invasive procedures, parenting after assisted conception, and adoption and infertilityrelated grief and shame $[31 \cdot, 32]$.

\section{New Procedures}

\section{Fertility Preservation}

Some believe that the emerging field of fertility preservation still necessitates regulation, especially given the significant rise in cancer survival rates. The argument is that the premature death of a cancer survivor should not factor into the decision to administer fertility treatments to cancer patients, because if the cancer patient is single, she may be in a stable relationship by the time she is ready to procreate. Furthermore, a self-selection process is likely, because women who are most likely to return for their stored genetic material are those who are healthy enough to withstand pregnancy [33]. Shah et al. [33] posit that insurance companies should be ethically obliged to fertility preservation because of impending iatrogenic damage to the reproductive organs, similar to existing policies that cover reconstructive breast surgery after mastectomy for breast cancer. The authors also suggest that women fertilize half of the embryos with their partner's sperm and the other half with donor sperm to avoid the potential problem of frozen embryo use in the chance that the couple separates. 


\section{Uterine Transplant}

In the past, UTx for women suffering from uterine factor infertility (UFI) was seen as unethical due to a lack of data on safety and efficacy. Lefkowitz and colleagues [34] propose newer, ethical criteria, because although a successful UTx leading to gestation has not yet been successful, recent investigations using animal models have shown promising results. The authors propose that more support for this procedure will grow given the increasing prevalence and acceptance of nonvital organ transplants, as well as the immunosuppression protocols after other organ transplants used for pregnant women that have shown minimal gestational harm. This results in an ethical debate on UTx between the principles of autonomy and nonmaleficence.

More complex ethical questions that will arise will be in the context of research. Informed consent may be problematic, because the subjects of UTx trials will be considered healthy research volunteers because UFI is a condition that does not require medical intervention to reduce morbidity or mortality. There also are dilemmas in the medical and psychological consequences of uterus donation wherein the hysterectomy literature shows problems with loss of gender identity and effects on sexuality [34]. Lefkowitz et al. [34] suggest that giving the potential donor comprehensive information, adequate time to consider the decision, psychological screening, follow-up, and ongoing care is the ethical approach. Moreover, other organ transplantation studies have noted the complex relationship that develops between a donor and recipient. In light of this, the authors suggest that it will be very important to safeguard the principle of anonymity of donor and recipient unless they have waived that right.

Furthermore, women in early trials should have low expectations of carrying and giving birth to a healthy baby, which might be hard for this group of women who are making an emotional decision to attempt to carry a pregnancy in an implanted uterus. Receiving a donated organ can have both physical and psychological risks, thus greater counseling may be needed to address issues, such as personal identity and bonding with the baby. Finally, the field of Reproductive Endocrinology and Infertility does not have policies to exclude women from treatment, yet transplantation does; thus, the two fields may have to reevaluate their policies in the future [34].

\section{Conclusions}

In this review article, we have looked at issues of ovarian stimulation, particularly HOMBs, egg banking (especially for research purposes), and diminished ovarian reserve. We also outlined an emerging body of literature on psychosocial distress, which includes greater attention to subgroups of infertile women, as well as counseling suggestions. Men have garnered more attention in the literature, either in their relationships with infertile women or as the infertility patient. Last, we outlined some ethical issues posed by newer procedures of fertility preservation and UTx.

Although the literature on ethical and psychosocial aspects of infertility spans from the biological, to the social, to the psychological, to the ethical, these issues all become intertwined in caring for the infertility patient. In sum, paying attention to psychosocial and ethical issues of infertility and fertility treatments makes for better practice, which is beneficial for practitioners and patients alike.

Acknowledgments Federal support for this study was provided by the Eunice Kennedy Shriver National Institute of Child Health \& Human Development (NICHD) as follows: Dr. Leyser-Whalen as an NRSA postdoctoral fellow under an institutional training grant (T32HD055163; Berenson, PI). The content is solely the responsibility of the authors and does not necessarily represent the official views of the NICHD or the National Institutes of Health.

The research reported in this manuscript was funded in whole or in part by NIH funding and is subject to the NIH public access policy, Division G, Title II, Section 218 of PL 110-161 (Consolidated Appropriations Act, 2008). The University of Texas Medical Branch at Galveston previously obtained nonexclusive rights in this manuscript that allow the final peer-reviewed manuscript to be submitted to the NIH upon acceptance for publication, including all modifications from the peer review process, to be made available to the public in PubMed Central as soon as possible but no later than 12 months after the official date of publication.

For further information, please contact the Center for Technology Development at (409) 772-7953.

Disclosure No potential conflicts of interest relevant to this article were reported.

\section{References}

Papers of particular interest, published recently, have been highlighted as:

- Of importance

1. Frith L, Jacoby A, Gabbay M. Ethical boundary-work in the infertility clinic. Soc Health Illness. 2011;33:570-85.

2. Li XH, Ma YG, Geng LH, et al. Baseline psychological stress and ovarian norepinephrine levels negatively affect the outcome of in vitro fertilization. Gyn Endocrin. 2011;27:139-43.

3. Paul-Simon A. Infertility and multiples. Newborn Infant Nurs Rev. 2011;11:180-4.

4. Beall SA, DeCherney A. History and challenges surrounding ovarian stimulation in the treatment of infertility. Fertil Steril. 2012;97:795-801.

5. Lee KC. Fertility treatments and the cost of a healthy baby. Nurs Womens Health. 2011;15:15-8.

6. Manninen BA. Parental, medical, and sociological responsibilities: "Octomom" as a case study in the ethics of fertility treatments. J Clinic Res Bioeth. 2011;S1:1-11. This article explores the ethical dimensions of fertility treatments that have potential to create 
higher-order multiple births. Ethical responsibility is placed on patients, physicians, insurance companies, and the media.

7. Hou X. Con: Should there be a limit of one single embryo implanted during an IVF cycle? MCN Am J Matern Child Nurs. 2011;36:153.

8. Huang Z. Pro: Should there be a limit of one single embryo implanted during an IVF cycle? MCN Am J Matern Child Nurs. 2011;36:152.

9. Kalfoglou AL, Sauer MV. A precautionary approach to oocyte donation for stem ell nuclear transplantation. Am J Bioethics. 2011;11:31-3.

10. Ellison B, Meliker J. Assessing the risk of ovarian hyperstimulation syndrome in egg donation: implications for human embryonic stem cell research. Am J Bioethics. 2011;11:22-30.

11. Batzer F, Daar J. Harmony and compensation for oocyte providers. Am J Bioethics. 2011;11:39-41.

12. Mertes H, Pennings G. Ethical concerns eliminated: safer stimulation protocols and egg banking. Am J Bioethics. 2011;11:33-5.

13. Fiore RN, Hinsch KM. Oocytes for research: reevaluating risks and compensation. Am J Bioethics. 2011;11:42-3.

14. Bamford R. Reconsidering risk to women: oocyte donation for human embryonic stem cell research. Am J Bioethics. 2011;11:379.

15. Kalbian A. Considering the risks to economically disadvantaged egg donors. Am J Bioethics. 2011;11:44-5.

16. Stein AL. The conundrum of oocyte donation, human research, OHSS, and ethics. Am J Bioethics. 2011;11:35-7.

17. Karipcin FS, Hossain A, Phelps JY. A legal-ethical analysis of reproductive endocrinologists' right to refuse ovulation induction to patients with diminished ovarian reserve. J Assist Reprod Genet. 2011;28:1105-9.

18. Greil AL, McQuillan J, Shreffler KM, et al. Race-ethnicity and medical services for infertility: stratified reproduction in a population-based sample of U.S. women. J Health Soc Behav. 2011;52:493-509.

19. Greil AL, Johnson KM, Mcquillan J, Lacy N. Are prior pregnancy outcomes relevant for models of fertility-specific distress or infertility help-seeking? Hum Fert. 2011;14:160-6.

20. McCarthy MP, Chiu S-H. Differences in women's psychological well-being based on infertility treatment choice and outcome. J Midwifery Women's Health. 2011;56:475-80.

21. Johnson KM, Fledderjohann J: Revisiting "her infertility: medicalized embodiment, self-identification and distress. Soc Sci Med, 2012, in press.
22. Greil AL, Shreffler KM, Schmidt L, McQuillan J. Variation in distress among women with infertility: Evidence from a population-based sample. Hum Reprod. 2011;26:2101-12.

23. Martins MV, Peterson BD, Almeida VM, Costa ME. Direct and indirect effects of perceived social support on women's infertilityrelated stress. Hum Reprod. 2011;26:2113-21.

24. Blyth E. Guidelines for infertility counseling in different countries: is there an emerging trend? Hum Reprod. 2012;0:1-12. doi:10.1093/humrep/des112.

25. Machin L. A hierarchy of needs? Embryo donation, in vitro fertilization and the provision of infertility counselling. Patient Ed Counsel. 2011;85:264-8.

26. Storrow RF. The ethics of exclusion in infertility care. J Clin Res Bioeth. 2011;2:114-5.

27. Palha AP, Lourenço MF. Psychological and cross-cultural aspects of infertility and human sexuality. Adv Psychosom Med. 2011;31:164-83.

28. Thompson EH, Woodward JT, Stanton AL. Moving forward during major goal blockage: Situational goal adjustment in women facing infertility. J Behav Med. 2011;34:275-87.

29. Bak CW, Seok HH, Song SH, et al. Hormonal imbalances and psychological scars left behind in infertile men. J Andro. 2012;33:181-9.

30. Chubak B, Thomas AJ. Ethical dilemmas in male infertility. In: Sabenegh ES, editor. Current clinical urology: Male infertility: Problems and solutions. New York: Springer Science; 2011. p. $125-8$.

31. - Fisher JRW, Hammarberg K. Psychological and social aspects of infertility in men: an overview of the evidence and implications for psychologically informed clinical care and future research. Asian J Androl. 2012;14:121-9. This article reviews studies on the psychosocial aspects of infertility in men. Despite diversity in conceptualization, design, setting, and data collection, there was consistency in findings.

32. Steuber KR, Solomon DH. Relational uncertainty, partner interference, and privacy boundary turbulence: explaining spousal discrepancies in infertility disclosures. J Soc Personal Relation. 2012;29:3-27.

33. Shah DK, Goldman E, Fisseha S. Medical, ethical, and legal considerations in fertility preservation. Intl $\mathrm{J}$ Gyn $\mathrm{Ob}$. 2011;115:11-5.

34. Lefkowitz A, Edwards M, Blayla J. The Montreal criteria for the ethical feasibility of uterine transplantation. Transplant Intl. 2012;25:439-47. 